\title{
Trizonal Speargun Injury Spanning Infratemporal, Transoropharyngeal and Transcervical Regions: A Case Report \\ P Brown, G Nicholson, G Channer
}

\begin{abstract}
Spear gun penetrating injury (SPGI) in the head and neck region albeit uncommon, can present with various challenges in management. We present a case of a male patient who sustained an intentional spear gun injury spanning infratemporal, transoropharyngeal and transcervical regions . The challenges and management outcomes of this case will be highlighted.
\end{abstract}

Keywords: Neck trauma, penetrating neck, speargun

Affiliation: Department of Surgery, Ear, Nose and Throat division, Kingston Public Hospital, Kingston, Jamaica.

Correspondence: Dr P Brown, Department of Surgery, Ear, Nose and Throat division, Kingston Public Hospital, North Street, Kingston, Jamaica. E-mail: drphillipbrown.ent@gmail.com

\section{INTRODUCTION}

Spear gun penetrating injury (SGPI) in the head and neck region can present with various clinical presentations ranging from mild to life threatening injuries depending on the spear's trajectory and associated structural damages. In addition, the spear has unique features such as a barbed 
end that once impacted can only be removed in an outward motion and therefore impacts surgical planning and removal. Simple retraction through its entry point can result in more tissue or life threatening injuries(1). Evaluation therefore portends early detection and treatment of life threatening issues such as airway or haemodynamic compromise. In a stable patient, radiological evaluation is of paramount importance in not only indicating path of injury but also to facilitate surgical planning and a multidisciplinary team approach. SGPI can occur accidentally; however, in our local setting as in this index case, it can be utilized as a weapon intentionally. We highlight our clinical approach, surgical challenges and the management outcomes.

\section{CASE REPORT}

A 32 year old male patient was referred from a peripheral hospital with a history of being shot with a fish spear gun by unknown assailants. There was a history of odynophagia and mild haemoptysis. There was no significant airway, neurovascular compromise or other digestive tract symptoms. Clinical examination revealed a fish spear with entry wound $5 \mathrm{~cm}$ anterior to the right tragus and $2 \mathrm{~cm}$ superior to the mid zygomatic arch, directed inferomedially approximately 60 degrees to the horizontal plane. It spanned the posterior oropharyngeal wall from a right superolateral to left inferior medial direction passing posterior to the left tonsil. The barbed end

tip exited the left neck at the posterior border of the left sternocleidomastoid muscle at the level of the hyoid bone (Fig. 1). 


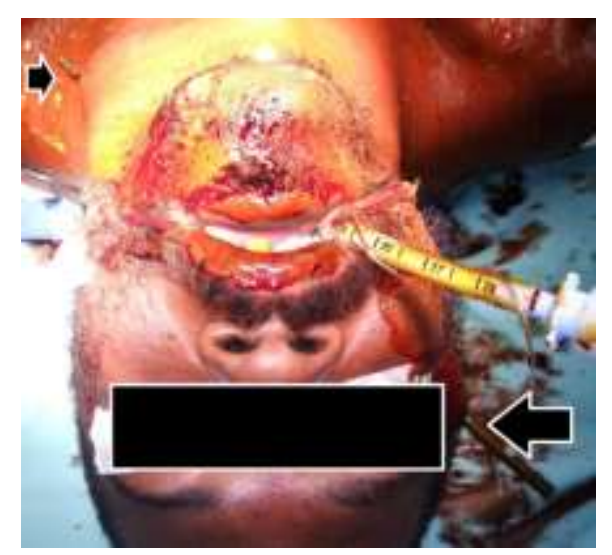

Fig. 1: Demonstrating Spear gun trajectory. Fish gun spear (Black arrows).

The left side of neck had evidence of localized surgical emphysema around the exit wound of the spear. The patient had no signs of neurovascular or other aerodigestive injuries. He had a CT Angiogram which demonstrated the course of the spear spanning infratemporal, transoropharyngeal and transcervical regions (Figures 2 and 3).There was associated surgical emphysema and no obvious significant vascular injury. The CTA views interpretation were however obscured by artefactual beams from the spear itself. A multidisciplinary team approach was undertaken comprising ENT (primary surgical team), Maxillofacial and Anaesthesia teams. 


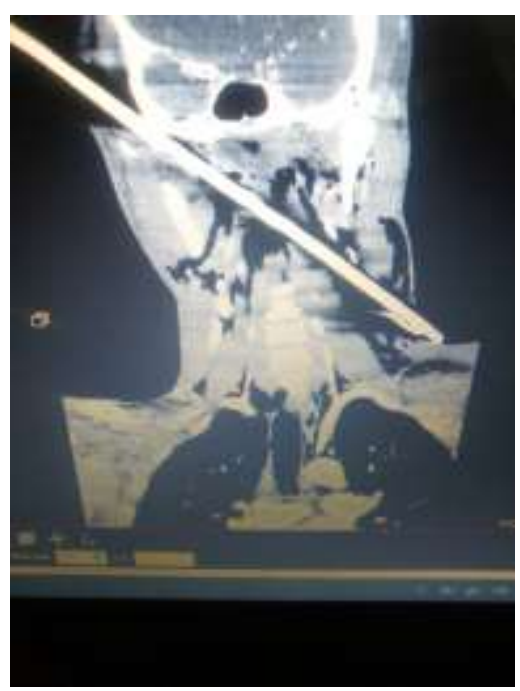

Fig. 2: demonstrating coronal views of spear spanning Infratemporal, transoropharyngeal and transcervical regions

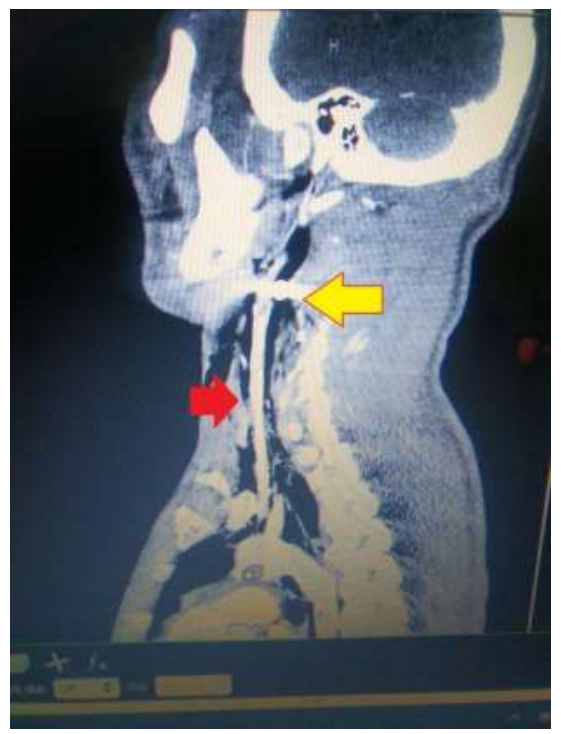

Fig. 3: demonstrating sagittal view of spear posterior relation to left common carotid artery Red arrow-common carotid artery Yellow arrow- spear 
Intraoperative findings revealed the spear spanning the infratemporal and oropharyngeal regions as previously described. In the neck, it passed posterior to the proximal internal and external carotid arteries and then through the internal jugular vein (IJV). There was no associated bleeding or haematoma as the spear had tamponaded the IJV transection injury. The spear then passed posterior to the left Spinal accessory nerve and exited the neck posterior to left sternocleidomastoid muscle (Figure 4). The IJV was ligated and the barbed end of the fish gun spear was pulled out the neck under direct vision with no significant sequelae (Figure 5). The oropharyngeal injury was repaired primarily and the maxillofacial injuries addressed electively. He was discharged five days postoperatively with no complications and last follow-up at three months was unremarkable.

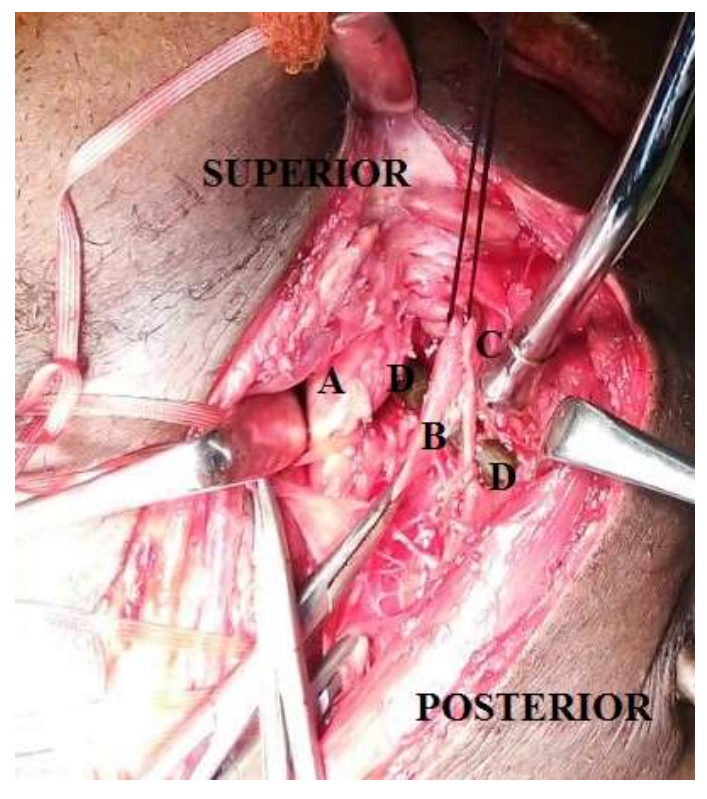

Fig. 4: Demonstrating intraoperative neck findings. A-proximal internal and external carotid arteries B- transected IJV C-Spinal Accessory D-fish spear gun. 


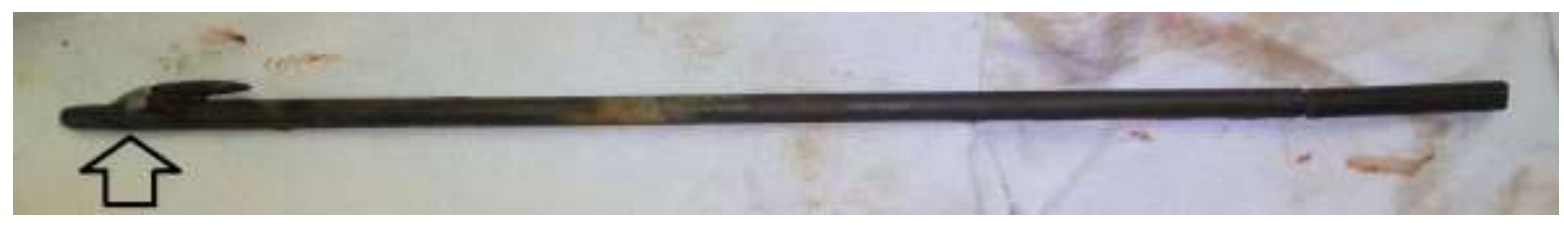

Fig. 5: demonstrating fish gun spear. Barbed end (open arrow)

\section{DISCUSSION}

Spear gun injuries in the head and neck region are uncommon and few reports (1-4) exist in the literature. These injuries pose unique clinical challenges due to specific and unusual features of the spear gun and the associated spear(1). The methodology employed in removal of a fish gun spear, as in this case, therefore requires understanding of the structures of these instruments and their mechanisms of operation(5). The spear gun designed for fishing, operates via a trigger mechanism with either compressed air or rubber band. Once activated, there is transmission of static energy to the spear to facilitate sudden acceleration(1). This design facilitates underwater hunting by overcoming water resistance (1). Therefore, devastating tissue injury can occur in the absence of water resistance accidentally or with intentional usage(1). In addition, there are several types of fish spear gun with different morphology. In the index case, the spear had a barbed end. This information facilitated and guided the appropriate surgical approach and precautions for removal.

The management of patients with SGPI, once immediate life threatening issues are addressed, is centered on determining the type, course, direction, entry and exit points of the spear. The relationship to the surrounding neurovasculature and aerodigestive tract is also of paramount importance. This is often employed with radiological investigations, notably a CT Angiogram as was done in this case. This will form the substratum for a multidisciplinary team 
approach and surgical planning. In the index case, this proved pivotal, for example, we were able to plan with Anaesthetic team, airway management issues and other possible required interventions. This also assisted in the surgical planning and contributed significantly to the favourable outcome in this patient.

The surgical planning for removal, addressing three different corridors in this case is predicated on standard surgical principles of wide exposure, vascular control, minimizing further tissue injury and minimal manipulation of the spear until final removal. This case had a unique finding of through and through injury of the IJV by the spear gun with no associated haeamtoma. This explained the paucity of clinical signs and radiological evidence of a significant vascular injury. This highlights the need to practice the standard surgical principles described above consistently to avoid significant morbidity and mortality.

\section{CONCLUSION}

Spear gun Injuries albeit uncommon pose unique clinical challenges upon presentation. Excellent outcomes are predicated on a number of factors. These include determining the type of spear and trajectory, relation to vital structures and addressing immediate life threatening injuries. This can be achieved with proper clinical and radiological evaluation. All of this will facilitate a multidisciplinary team approach which can prove pivotal in surgical planning and achieving favourable outcomes. This was accomplished in our index case by applying the above principles despite the challenge of a spear spanning three corridors in the head and neck region. 


\section{AUTHORS' NOTE}

P Brown conceived the paper, wrote the manuscript and approved the final version. G Channer and G Nicholson edited and approved the final version. 


\section{REFERENCES}

1. Alper M, Totan S, Cankayali R, Songur E. Maxillofacial spear gun accident: report of two cases. J Oral Maxillofac Surg 1997; 55: 94-7.

2. Bakhos D, Villeneuve A, Kim S, Lebrun H, Dufour X. Head Spear Gun Injury: An Atypical Suicide Attempt. J Craniofac Surg 2015; 26: e547-8.

3. Mamadi P, Seta W. Through-and-through penetrating spear gun injury of the head: case report. P N G Med J 2007; 50: 74-6.

4. Ban LH, Leone M, Visintini P, Blasco V, Antonini F, Kaya JM, et al. Craniocerebral penetrating injury caused by a spear gun through the mouth: case report. J Neurosurg. 2008; 108: 1021-3.

5. Sadda RS. Fish-gun injury of the maxillofacial region. J Oral Maxillofac Surg. 1996 ; 54: $1132-5$. 